\title{
Bio-inspired smart surface to achieve controllable locomotion through adjustable anisotropic friction
}

\author{
Zhongying JI ${ }^{1}$, Shiyu QIN ${ }^{2}$, Shuanhong MA ${ }^{1}$, Xin JIA ${ }^{2}$, Xiaolong WANG ${ }^{1, *}$, Feng ZHOU ${ }^{1}$ \\ ${ }^{1}$ State Key Laboratory of Solid Lubrication, Lanzhou Institute of Chemical Physics, Chinese Academy of Sciences, Lanzhou 730000, China \\ ${ }^{2}$ School of Chemistry and Chemical Engineering, Shihezi University, Shihezi 832003, China \\ Received: 13 June 2020 / Revised: 17 October 2020 / Accepted: 15 April 2021 \\ (C) The author(s) 2021.
}

\begin{abstract}
Anisotropic friction generated by microstructured surfaces is crucial for performing functions such as directional locomotion and adhesion in biological systems. Hence, an epoxy-based shape memory polymer (SMP) incorporating $\mathrm{Fe}_{3} \mathrm{O}_{4}$ nanoparticles is used in this study to create a smart surface with oriented structures to mimic anisotropic friction and exploit human-developed controllable locomotion systems. Applying the specific properties of the epoxy-based SMP, fast switching friction can be achieved by adjusting the topography and stiffness of the microstructures on the surface. In addition, the photothermogenesis effect of $\mathrm{Fe}_{3} \mathrm{O}_{4}$ nanoparticles induces changes in the asymmetric topography and stiffness on the SMP surface under the irradiation of near-infrared (NIR) light, thereby inducing a rapid switching of the friction force. Furthermore, a microbot is created to demonstrate remotely controlled locomotion, such as unidirectional and round-trip movements, and braking by switching the friction force under NIR light. These results are promising for the design of new intelligent surfaces and interfaces; additionally, they may facilitate the investigation of biological structures and processes.
\end{abstract}

Keywords: friction; smart surface; shape memory polymer (SMP); remote control

\section{Introduction}

Anisotropic friction is ubiquitous in nature, and asymmetric forces must generate to achieve adhesion, locomotion, and transportation [1-5]. Examples of anisotropic friction include the highly ordered fibers of the snake skin [1], which exhibit a pronounced anisotropic friction force to propel its locomotion, i.e., high friction for propulsion and low friction for sliding. Complicated physical phenomena and processes may be involved in natural anisotropic friction systems; however, in many cases, a typical phenomenon encountered in nature is anisotropic microstructures on surfaces that are oriented at a certain angle to the supporting layer. It has been discovered that the anisotropic friction force generated from asymmetric microstructures primarily depends on two dominant factors: the surface topography and the stiffness of the biological surface [6]. As one of the most effective topographies for generating anisotropic friction force, hook-like spines are ubiquitous in various plants and animals, such as wheat awn, Galium aparine, fruit of Xanthium L, filefish skin. [7-9]. In addition, the stiffness of the biological surface, which has not been investigated extensively previously, has recently demonstrated its discrepant self-adaption and friction force properties $[9,10]$.

Various artificial surfaces that imitate anisotropic microstructures have been employed for smart actuation, soft robots, surface engineering, and medical devices [11-18]. However, most surfaces inspired by single natural microstructures demonstrate constant stiffness

* Corresponding author: Xiaolong WANG, E-mail: wangxl@licp.cas.cn 
and isotropic or anisotropic friction behaviors. Therefore, new strategies are required for the development of smart materials to achieve reversible transitions between isotropic and anisotropic friction forces. Although frictional anisotropy allows the fabrication of smart devices for applications ranging from directional driving to cargo transportation, challenges remain, such as the speed of real-time control, low-efficiency locomotion, and single-directional movement, which affect the performance and potential applications of smart devices.

Previously, the studies focused on achieving an anisotropic friction force and locomotion by controlling the stiffness and orientation of biomimetic surfaces [7-9]. However, shape change is a passive process under an external force, and the switched angle of an oriented structure is limited. Inspired by the typical asymmetric microstructure of a hook-like spine, which is one of the most typical and effective features of natural systems, a smart surface was built via three-dimensional (3D) printing and replicating using an epoxy-based shape memory polymer (SMP) incorporating $\mathrm{Fe}_{3} \mathrm{O}_{4}$ nanoparticles $\left(\mathrm{Fe}_{3} \mathrm{O}_{4} \mathrm{NPs}\right)$ to mimic the locomotion behavior of biological systems induced by adjustable anisotropic friction. SMPs have been widely reported to be a smart material for dynamically controlling surface microstructures owing to its shape memory performance, particularly in the field of surface wetting [19-21], whereas the application of SMPs in the field of asymmetric surface microstructures for investigating friction has rarely been reported. Furthermore, epoxy-based SMPs show a softening effect once placed in a high-temperature atmosphere, and this softening effect results in the disappearance of the anisotropic friction behavior of the biomimetic surface, as previously reported in Ref. [6]. Therefore, by exploiting the photothermogenesis of $\mathrm{Fe}_{3} \mathrm{O}_{4}$ NPs and the shape memory property of SMPs, a surface with a switchable friction force was fabricated by synergistically changing the orientation and stiffness using near-infrared (NIR) light as a trigger. Moreover, the paradigm of a microbot prototype was implemented to perform directionality, fast and controllable locomotion, and round-trip movements stimulated by NIR light. The concepts of dynamic friction control and biomimetic smart surfaces will provide a general yet effective construction concept for the development of new actuators, sensors, and robots.

\section{Experimental}

\subsection{Materials}

Diglycidyl ether of bisphenol A-type epoxy resin (E51) was purchased from Baling Petroleum \& Chemical Corporation, China. The curing agents, m-xylenediamine (MXDA) and n-octylamine (OA), were purchased from Energy Chemical, Shanghai, China. Polydimethylsiloxane (PDMS-Sylgard 184), which was used as a template, and $1 \mathrm{H}, 1 \mathrm{H}, 2 \mathrm{H}, 2 \mathrm{H}-$ perfluorooctyltriethoxysilane, which was used as a fluoride molecule, were obtained from J\&K Chemical Ltd., China. $\mathrm{Fe}_{3} \mathrm{O}_{4}$ NPs were synthesized based on previously reported procedures [22, 23]. The contacting pair of the PDMS hemisphere was prepared by curing the precursor with a curing agent at a ratio of 10:1 by weight in a 96-well confocal plate.

\subsection{Design and preparation of $3 \mathrm{D}$ printed biomimetic surfaces}

A solid 3D model of a biomimetic surface with a bottom diameter $(d)$ of $\sim 480 \mu \mathrm{m}$, height $(h)$ of $\sim 880 \mu \mathrm{m}$, radius $(r)$ of $\sim 1,000 \mu \mathrm{m}$, and distance (s) of $\sim 750 \mu \mathrm{m}$ between the spines was designed using SolidWorks based on a morphology inspired by natural systems (Fig. 1(a)). Subsequently, the designed surface with a sample size of $20 \mathrm{~mm} \times 20 \mathrm{~mm}$ was built using a digital light processing (DLP) 3D printer (CR-6 from Chuangxiang 3D Ltd., Shenzhen, China) by photopolymerizing an acrylate-based liquid resin in a layer-by-layer sequence. Once completed, the 3D printed samples were washed in ethanol, followed by post-curing with ultraviolet light.

\subsection{Fabrication of $\mathrm{SMP} @ \mathrm{Fe}_{3} \mathrm{O}_{4}$ surfaces}

A simple template method was employed to fabricate $\mathrm{SMP} @ \mathrm{Fe}_{3} \mathrm{O}_{4}$ surfaces. To separate the template from the sample, every negative template was first modified with $1 \mathrm{H}, 1 \mathrm{H}, 2 \mathrm{H}, 2 \mathrm{H}$-perfluorooctyltriethoxysilane in a decompressed environment at room temperature for $24 \mathrm{~h}$ and then heated at $80^{\circ} \mathrm{C}$ for $3 \mathrm{~h}$. The PDMS precursor was mixed with a curing agent at a weight 


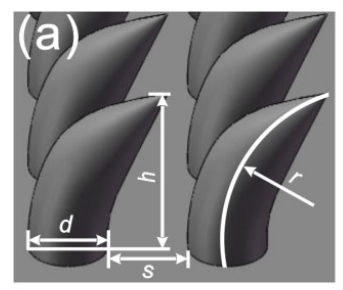

(d)

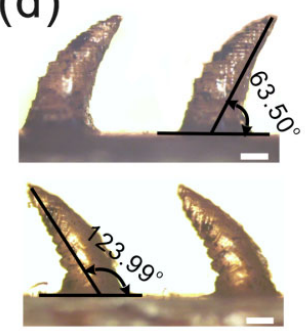

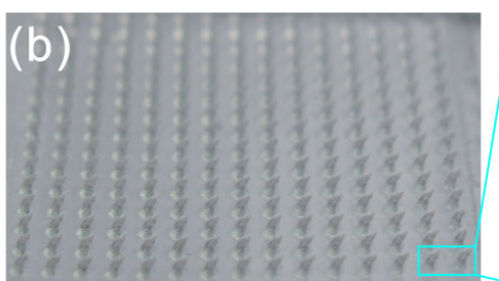



(e)

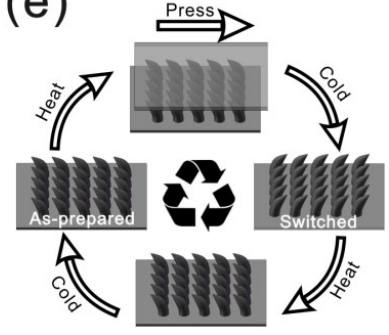

(f)

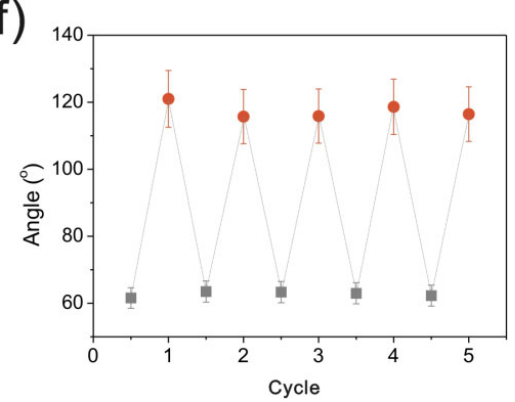

Fig. 1 (a) Characteristics of spines on biomimetic surface; (b, c) digital and scanning electron microscopy (SEM images of fabricated surface with anisotropic spines, respectively (scale bar $=200 \mu \mathrm{m}$ ); (d) as-prepared (upper) and permanent orientation of asymmetric spines; switched (lower) and temporary orientation of spines; (e) preparation process for as-prepared and switched surfaces and their mutual conversion; (f) reversible variation of spines through repeated pressing and recovery at $100{ }^{\circ} \mathrm{C}$, with the process repeated five times using a sample measuring $20 \mathrm{~mm} \times 5 \mathrm{~mm}$ (the values are expressed as mean \pm standard deviation).

ratio of 10:1 and placed in a centrifuge to remove air bubbles (3,000 rpm, $5 \mathrm{~min})$; subsequently, it was poured onto the 3D printed modified surface. As shown in Fig. S1 in the Electronic Supplementary Material (ESM), after precursor heating at $70^{\circ} \mathrm{C}$ for $4 \mathrm{~h}$, the PDMS negative template was peeled off slowly along the orientation of the hook-like spines to preserve the microstructures. Finally, an SMP mixture comprising E51, MXDA, and OA at a molar ratio of 4:1:2, and $\mathrm{Fe}_{3} \mathrm{O}_{4}$ NPs of $0.5-3.0 \mathrm{wt} \%$ were added to the fluorinated PDMS negative template and then cured at $60^{\circ} \mathrm{C}$ for $4 \mathrm{~h}$. After peeling off the cured epoxy-based composite from the PDMS template, a surface with oriented structures was obtained (referred to herein as the "as-prepared surface"). In addition, to obtain a surface with switched spines, the as-prepared surface was first heated at $100{ }^{\circ} \mathrm{C}$ for approximately $8 \mathrm{~min}$, after which it was pressed using a pre-cleaned glass slide under a specified pressure $(\sim 0.098 \mathrm{~N}$, Fig. S2 in the ESM). Subsequently, the surface was cooled to room temperature under the same pressure, after which switched microstructures were formed (resulting in a "switched surface") [19, 20]. To recover the switched surface, it was reheated at $100{ }^{\circ} \mathrm{C}$ for approximately $30 \mathrm{~s}$ in an oven, during which the switched microstructures resumed to their initial state.

\subsection{Friction tests}

In the experiment performed in the present study, friction measurements were conducted on a 14-FW static-dynamic tribometer (HEIDON Co., Ltd., Japan) with sphere-to-flat contact and in the single sliding direction mode to record the friction force (Figs. S3(a) and S3(b) in the ESM). The samples tested (measuring $20 \mathrm{~mm}$ (length) $\times 5 \mathrm{~mm}$ (width) $\times 1.5 \mathrm{~mm}$ (thickness)) were fixed tightly onto a testing platform, and an elastic PDMS hemisphere (with a diameter of $6 \mathrm{~mm}$ and an elastic modulus of $1.8 \mathrm{MPa}$ ) was employed as the contacting pair with a sliding distance of $20 \mathrm{~mm}$ and a sliding velocity of $20 \mathrm{~mm} \cdot \mathrm{min}^{-1}$. To investigate the dynamic friction control, an NIR laser light spot (with a diameter of $5 \mathrm{~mm}$ and a wavelength of $808 \mathrm{~nm}$, produced by Lasever Inc. of China and with Dpssl Driver II power) was moved over the surface at $1.0 \mathrm{~mm} / \mathrm{s}$ during the friction measurements. The friction forces of the tribopair sliding along and against the topographic orientation of the microstructures were defined as the positive and negative friction forces, respectively (Fig. S4 in the ESM) [8]. The quantitative characterization of the frictional anisotropy was determined by the friction force difference, which is the absolute value of the difference between the friction 
forces in the negative and positive sliding directions [8]. Notably, for the friction tests without NIR irradiation, friction measurements were performed five times for each specimen. When the friction measurements were performed under NIR light, each specimen could only be measured once, and this procedure was repeated five times using different specimens. This is because the surface morphology after measurement changed because of the synergistic effect of the normal load in the sliding process and the softening effect under NIR light irradiation.

\subsection{Demonstration of vibration-assisted smart driving}

An ultrasonic cleaner (KQ-5200, Kunshan Ultrasonic Instruments Co., Ltd., China) with an ultrasonic frequency of $40 \mathrm{kHz}$ and an ultrasonic power of $200 \mathrm{~W}$ was used as the vibrating platform. A biomimetic microbot with shape memory microstructures was placed on a vibrator with oriented spine structures facing down. During braking, a pair of tweezers was used to decelerate and halt the motion of the microbot because the single softening effect stimulated by the NIR light could not stop the device immediately in a short time. In the return process, only NIR light was used to switch the orientation of the spine structure and trigger the microbot to drive in the opposite direction. In contrast to the partial exposure of the surface to NIR light during the friction measurement, the entire sample was irradiated during the vibrationassisted driving process. This is because the former process was performed to investigate the friction control results from the spine switching, whereas the latter process was performed to obtain a higher speed and an easier control of the entire sample.

\subsection{Characterization}

The surface morphologies were characterized using the scanning electron microscope (SEM, JSM-5600LV, Japan) at $20 \mathrm{kV}$ and an optical microscope (Olympus BX51, Japan). The glass transition temperature and storage modulus were evaluated via dynamic mechanical analysis (DMA 242E from Netzsch GmbH, Germany) at a heating rate of $3 \mathrm{~K} \cdot \mathrm{min}^{-1}$. The mechanical properties of the samples were evaluated using an electrical universal material testing machine (EZ-Test,
Shimadzu, Japan). Statistical product and service solutions (SPSS 20.0, International Business Machines Corporation, USA) with Fisher's F-test were used for the statistical analyses of different friction forces, and the statistical significance was defined as ${ }^{* *} P<0.01$.

\section{Results and discussion}

\subsection{Design and fabrication of biomimetic shape memory surface with anisotropic microstructures}

In nature, various geometries of surface topography exist for different functions. In anisotropic friction, mechanical interlocking, and locomotion behaviors, the asymmetric geometry of a hook-like spine is one of the most typical features owing to its typical and effective properties that induce frictional anisotropy [7-9, 24-26]. Therefore, the hook-like spine morphology is observed in wheat awn, fruit of Xanthium L, hind leg of cricket, and skin of filefish (as shown in Figs. S5(a)-S5(d) in the ESM, respectively); it exhibits the coincident orientation of microstructures, which enables the production of anisotropic friction. Hence, a surface coated with highly ordered hook-like spines was designed and used in this study to mimic controllable locomotion behaviors based on the adjustable anisotropic friction induced by the switching of the spine orientation and surface stiffness (Figs. 1(a) and S5(e) in the ESM). A surface with hook-like spines was built using a DLP-based 3D printing technique. The digital image in Fig. 1(b) shows that the printed surface was covered with hook-like spines exhibiting the same orientation and morphology. The SEM image in Fig. 1(c) shows the printed features at the connection between the spines and the substrate. An epoxybased SMP with an apparent thermal reversible phase transition ability was used to duplicate the biomimetic surface. Such a surface possesses excellent shape memory ability and allows the memorization of the different orientations of the microstructures [19, 20], thereby resulting in various frictional performances. The biomimetic surface with a permanent shape (with an incline angle to the horizontal substrate of $63.5^{\circ}\left( \pm 3.1^{\circ}\right)$, see the upper image in Fig. $\left.1(\mathrm{~d})\right)$ was heated to $100{ }^{\circ} \mathrm{C}$, which afforded high mobility in the molecular chain, and the modulus of the polymer decreased immediately, thereby causing a change in 
the thermodynamically stable state of the molecular chains [27]. An external pressure created using a glass slide was applied to the spines until an opposite orientation was achieved. Subsequent cooling under an external force below the glass transition temperature $\left(T_{\mathrm{g}}\right)$ caused the spines to remain in the opposite orientation (with an incline angle to the horizontal substrate of $124.0^{\circ}\left( \pm 7.42^{\circ}\right)$, see the lower image in Fig. 1(d)), thereby forming a "switched surface". After further heating at $\sim 100{ }^{\circ} \mathrm{C}$ for approximately $30 \mathrm{~s}$ in a free state, the switched spines were restored to their original configuration, and their initial state was preserved (Fig. 1(e)). Exploiting the shape memory ability, such a morphological transition of spines between the as-prepared and switched states can be achieved by changing the temperature. Furthermore, this epoxy-based SMP surface exhibits fatigue durability owing to its 3D crosslinking networks [19]. As depicted in Fig. 1(f), the orientation of the spines can be switched reversibly for at least five cycles without any deterioration, after which irreversible damage will occur between the spines and the substrate if the bending process is continued (Fig. S6 in the ESM).

\subsection{Friction measurement under different conditions}

The anisotropic friction force of a biomimetic surface under different conditions was investigated. As shown in Fig. 2(a), when the normal load was increased


Fig. 2 (a) Friction force of as-prepared and switched surfaces in positive sliding direction (above horizontal line) and negative sliding direction (below horizontal line) with a normal load from 0.1 to $0.9 \mathrm{~N}$; (b) typical friction signals of as-prepared and switched surfaces in positive and negative sliding directions at room temperature with $0.5 \mathrm{~N}$ load; (c) friction force in positive (gray) and negative (red) sliding directions at $80{ }^{\circ} \mathrm{C}$; (d) comparison of friction forces in negative sliding direction for 25 and $80{ }^{\circ} \mathrm{C}$; (e, f) typical friction signals for surface in positive and negative sliding directions, respectively, with $0.5 \mathrm{~N}$ load at $80{ }^{\circ} \mathrm{C}$. All friction measurements shown in Figs. $2(\mathrm{a})$, 2 (c), and 2(d) were repeated five times, and the friction force values are expressed as mean $\pm \operatorname{standard~deviation~}\left(n=5 ;{ }^{* *} p<0.01\right)$. 
from 0.1 to $0.9 \mathrm{~N}$, the friction force of the negative sliding direction was always greater than that of the positive sliding direction for both the as-prepared and switched surfaces. For example, for the as-prepared surface, the friction force difference (i.e., the difference between the friction forces in the positive and negative sliding directions) was $0.1 \mathrm{~N}( \pm 0.03 \mathrm{~N})$ at a normal load of $0.1 \mathrm{~N}$, whereas it increased to $1.51 \mathrm{~N}( \pm 0.08 \mathrm{~N})$ at a normal load of $0.9 \mathrm{~N}$, indicating that the surface with oriented structures exhibited outstanding loaddependent frictional anisotropy. In addition, the as-prepared and switched surfaces exhibited almost identical friction force values in both the positive and negative sliding directions, suggesting that the spines covering the switched surface possessed an almost identical topography to that of the spines covering the as-prepared surface. The typical friction signals shown in Fig. 2(b) further support this conjecture. For the same sliding direction, the curves possessed not only similar friction force values, but also analogous force fluctuations that originated from the discontinuous spines. Briefly, both the as-prepared surface and switched surfaces exhibited a uniform topography and evident frictional anisotropy.

Moreover, an SMP is a type of material that enables switching between a glassy state and a rubbery state via temperature change, and the elastic modulus decreases by two to three orders of magnitude once the thermal environment reaches the phase transition temperature [28]. Our previous study revealed that the friction force is dependent on the rigidity of the substrate used to support the microstructures of the surface [9]. Therefore, dynamic friction control of the biomimetic SMP surface can be readily achieved by changing the temperature and switching the orientation. Prior to the present study, the friction switching behavior of the SMP surface and its friction force in a rubbery state at $80{ }^{\circ} \mathrm{C}$ (higher than the glass transition temperature $T_{\mathrm{g}}$, see Fig. S7 in the ESM) were used for comparison. Evidently, when the normal load was increased from 0.1 to $0.9 \mathrm{~N}$, the friction force increased from $0.12 \mathrm{~N}( \pm 0.006 \mathrm{~N})$ to $0.53 \mathrm{~N}( \pm 0.03 \mathrm{~N})$ in the positive direction, and from $0.09 \mathrm{~N}( \pm 0.005 \mathrm{~N})$ to $0.56 \mathrm{~N}$ $( \pm 0.03 \mathrm{~N})$ in the negative direction, which is consistent with the friction properties at room temperature (a)

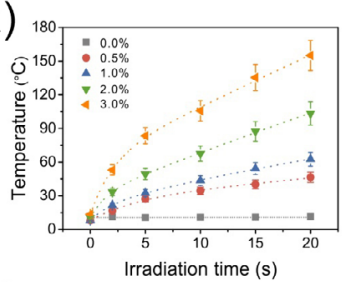

(b)
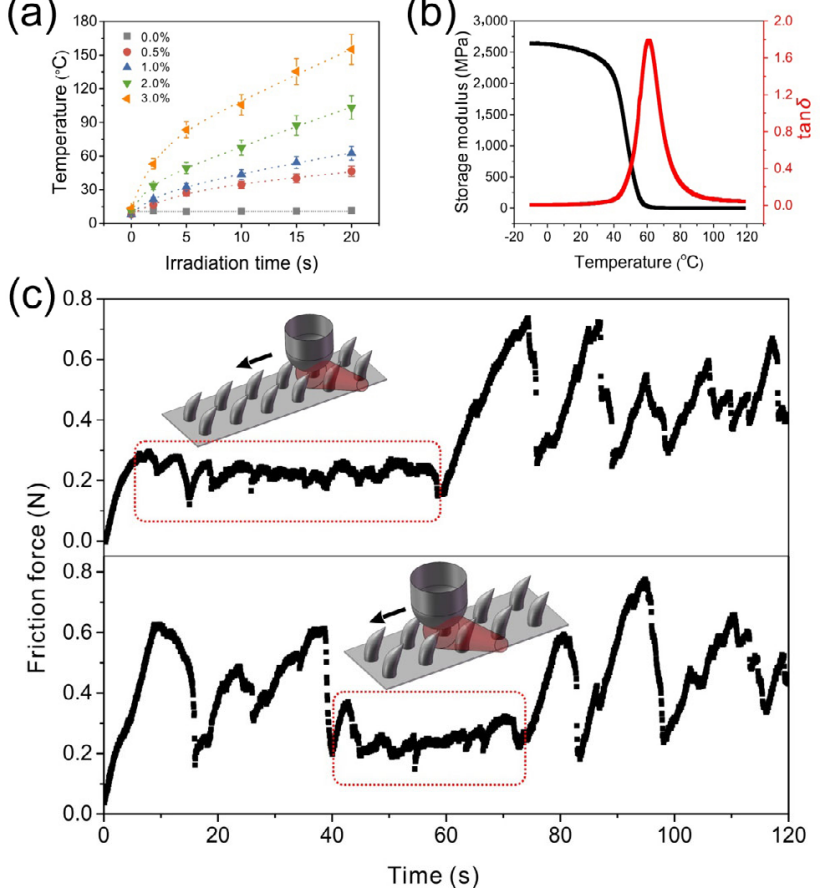

Fig. 3 (a) Temperature change with NIR irradiation time of SMP containing different $\mathrm{Fe}_{3} \mathrm{O}_{4}$ NPs; measurements were repeated five times using a sample measuring $20 \mathrm{~mm}$ (length) $\times 5 \mathrm{~mm}$ (width) $\times$ $2 \mathrm{~mm}$ (thickness); (b) storage modulus and loss factor $(\tan \delta$ ) curves of SMP@ $\mathrm{Fe}_{3} \mathrm{O}_{4}$ composite as a function of temperature; (c) changes in friction signals with NIR irradiation at the beginning of sliding (upper panel) and in the middle of sliding (lower panel) (with $0.5 \mathrm{~N}$ load in the negative sliding direction).

$\left(\sim 25^{\circ} \mathrm{C}\right)$. However, compared with the friction properties at room temperature, with a normal load of $0.9 \mathrm{~N}$ at $80^{\circ} \mathrm{C}$, for example, the friction force in the positive direction only increased slightly, whereas that in the negative direction decreased substantially from $1.74 \mathrm{~N}( \pm 0.08 \mathrm{~N})$ to $0.56 \mathrm{~N}( \pm 0.03 \mathrm{~N})$ (Fig. 2(d)). This phenomenon will result in the friction force exhibiting similar values in both the positive and negative sliding directions, i.e., the frictional anisotropy of the mollescent SMP surface will disappear (Fig. 2(c)). This might be because the flexible spines had larger contact areas during positive sliding, resulting in a greater friction force; meanwhile, during negative sliding, the flexible spines deformed easily owing to the sliding of the tribopair, thereby resulting in a lower friction force. The typical friction signals shown in Figs. 2(e) and 2(f) support this conjecture. The relatively smooth friction signals obtained in the negative sliding direction was a distinct phenomenon, even though force fluctuation 
occurred. Based on statistical analysis, it can be concluded that the friction force was sensitive $(p<0.05)$ to the temperature in the negative sliding direction (Fig. 2(d)), insensitive $(p>0.05)$ to the morphology between the as-prepared and switched surfaces (Fig. 2(a)), and insensitive to the sliding directions when the temperature increased to $80^{\circ} \mathrm{C}$ or higher (Fig. 2(c)).

\subsection{Friction switching behavior of biomimetic surface induced by rigidity and orientation}

The incorporation of $\mathrm{Fe}_{3} \mathrm{O}_{4}$ NPs can endow the SMP surface with an excellent NIR photothermogenesis effect $[29,30]$ for regulating the in situ interface temperature, thereby enabling a fast switching of friction force. First, the temperature increase traces of the as-prepared surface with different $\mathrm{Fe}_{3} \mathrm{O}_{4} \mathrm{NP}$ contents vs. the irradiation time were investigated, as shown in Fig. 3(a). Under a continuous NIR laser irradiation, the temperature of the SMP sheet incorporating $\mathrm{Fe}_{3} \mathrm{O}_{4} \mathrm{NPs}$ (the $\mathrm{SMP} @ \mathrm{Fe}_{3} \mathrm{O}_{4}$ sheet) exhibited a considerable increase within seconds, whereas no significant increase was observed in the pure SMP sheet. In addition, a greater doping amount of $\mathrm{Fe}_{3} \mathrm{O}_{4} \mathrm{NPs}$ resulted in a faster temperature increase. Specifically, when the $\mathrm{SMP} @ \mathrm{Fe}_{3} \mathrm{O}_{4}$ sheet had a doping amount of $3.0 \mathrm{wt} \%$, the surface temperature reached $\sim 83{ }^{\circ} \mathrm{C}$ with NIR laser irradiation lasting only $5 \mathrm{~s}$, indicating a vigorous photothermal performance. Furthermore, the $T_{\mathrm{g}}$ of the $\mathrm{SMP} @ \mathrm{Fe}_{3} \mathrm{O}_{4}$ sheet was evidenced by the $\tan \delta$ peak temperature, $\sim 61{ }^{\circ} \mathrm{C}$ (Fig. 3(b)) [19], illustrating that the SMP sheet and the $\mathrm{SMP} @ \mathrm{Fe}_{3} \mathrm{O}_{4}$ sheet had similar thermal systems (Fig. $\mathrm{S} 8$ in the ESM).

As expected, the SMP@ $\mathrm{Fe}_{3} \mathrm{O}_{4}$ surface was heated rapidly under NIR laser irradiation and hence acquired a lower friction force owing to the mollescent effect [9]. To validate this, the friction behavior of the $\mathrm{SMP} @ \mathrm{Fe}_{3} \mathrm{O}_{4}$ surface covered with hook-like spines was investigated by switching the NIR laser on and off, as shown in Fig. 3(c). Based on the case with the negative sliding direction as an example, the $\mathrm{SMP} @ \mathrm{Fe}_{3} \mathrm{O}_{4}$ surface exhibited a lower friction force $(\sim 0.26 \mathrm{~N})$ under NIR laser irradiation, and an abrupt increase occurred once the NIR light was turned off $(\sim 0.73 \mathrm{~N})$ (see the upper panel of Fig. 3(c)). The friction signals showed a relatively smooth line under NIR irradiation, similar to the signals for sliding in the positive direction, whereas a typical fluctuation appeared once the irradiation was turned off, which is consistent with the dynamic control at different temperatures (room temperature or $80{ }^{\circ} \mathrm{C}$ ). Interestingly, with its ability in providing rapid photothermogenesisinduced flexibility, the $\mathrm{SMP} @ \mathrm{Fe}_{3} \mathrm{O}_{4}$ surface enables the creation of a "real-time" and "free-area" friction switch. Figure 3(c) (see the lower panel) shows that when the tribopair passed over the spines in the negative direction, the friction force decreased abruptly from $\sim 0.63$ to $\sim 0.2 \mathrm{~N}$ once the NIR laser was turned on, and then increased to $\sim 0.58 \mathrm{~N}$ once the NIR laser was turned off. This phenomenon further demonstrates that the frictional anisotropy and friction behavior can be regulated with a "real-time" and "free-area" approach through a simple yet efficient NIR photothermogenesis strategy.

The temperature increase induced by NIR laser irradiation changed both the rigidity of the $\mathrm{SMP} @ \mathrm{Fe}_{3} \mathrm{O}_{4}$ surface and the orientation of its spines (Fig. S3(c), S9, and Video S1 in the ESM). Consequently, the switched surface with bent hook-like spines (switched by $\sim 180^{\circ}$ ) can obtain a tunable friction force by changing the orientation of the $\mathrm{SMP} @ \mathrm{Fe}_{3} \mathrm{O}_{4}$ spines. For comparison, a switched surface with as-prepared spines on the first half and bent spines on the second half was measured at room temperature, as shown in Fig. 4 (see the upper panel). When the tribopair slid from a positive to a negative area, the friction force increased abruptly (from $\sim 0.25$ to $\sim 0.61 \mathrm{~N}$ ) because the flexible PDMS ball was penetrated by the hard and peaked spines during negative sliding. Subsequently, NIR laser irradiation was employed to alter the friction force of the switched surface with bent spines, and the results for the friction behavior are shown in Fig. 4 (see the lower panel). The friction signals as a function of time exhibited a typical smooth line until $60 \mathrm{~s}$; however, a high friction force occurred once the NIR light was turned on, causing the bent spines to resume to their initial morphology, with the sliding direction changing from positive to negative. Unexpectedly, the increase in the velocity of the friction force was relatively slow compared with those of previous cases, even though the orientation of the spines switched completely. This can be attributed 


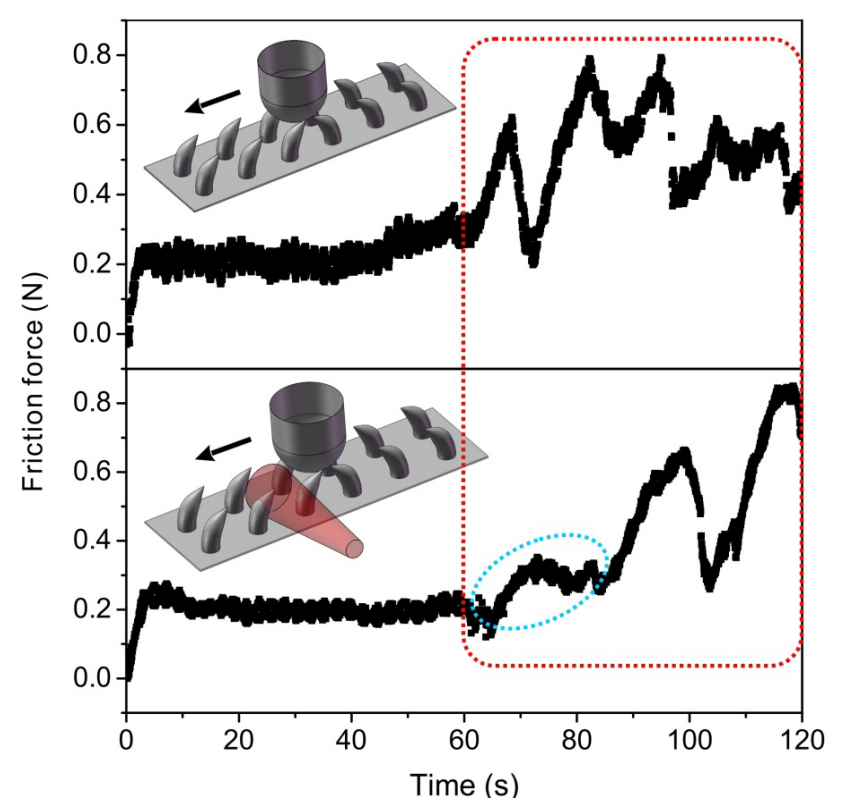

Fig. 4 Changes in friction signals without NIR irradiation (upper) and with NIR irradiation (lower) in negative sliding direction with $0.5 \mathrm{~N}$ load.

to the mollescent effect of the SMP and the effect of switching the spine orientation and decreasing the modulus of the SMP@ $\mathrm{Fe}_{3} \mathrm{O}_{4}$ surface during the NIR irradiation. In other words, the switched spines stored the energy produced by the NIR light, thereby resulting in the initial sliding (see the dashed blue circle in Fig. 4) on a flexible surface. By contrast, when the $\mathrm{SMP} @ \mathrm{Fe}_{3} \mathrm{O}_{4}$ surface resumed to room temperature, the friction force increased to a normal value $(\sim 0.59 \mathrm{~N})$. In contrast to the friction force of the as-prepared surface induced by a single element of rigidity, the friction force of the switched surface was affected by both the rigidity and the spine orientation, and the synergistic effect was primarily reflected in the negative sliding direction as many influencing factors were present, such as the contact area in the positive sliding direction.

\subsection{Bio-inspiration for directional driving, con- trollable braking, and free returning}

Directional driving, based on frictional anisotropy, is widespread in many systems $[1,7,9,14]$. The $\mathrm{SMP} @ \mathrm{Fe}_{3} \mathrm{O}_{4}$ surface created in the present study was inspired by such systems and possessed special shape memory ability, through which it can imitate anisotropic microstructures. This surface can potentially be used for controllable locomotion, including directional driving, controllable braking, and free return. Although a significant number of such functional surfaces have been used for directional actuation [9], transporting cargoes [7], and crossing a steep obstacle [13], most of them have demonstrated only one-way driving and are barely capable of braking or returning freely and/or continuously. In the present study, exploiting the excellent photothermal conversion and shape memory abilities of a bio-inspired smart surface, a biomimetic microbot prototype was developed and used for directional driving, controllable braking, and free return. In a demonstration, a microbot with an as-prepared $\mathrm{SMP} @ \mathrm{Fe}_{3} \mathrm{O}_{4}$ surface achieved directional driving at a speed of $\sim 25 \mathrm{~mm} / \mathrm{s}$ under vibration, in a direction opposite to that of the spines. Nevertheless, it only achieved unidirectional movement, and a U-turn can only be performed manually (Fig. 5(a) and Video S2 in the ESM). By contrast, a microbot with a switched surface is more functional and can achieve controllable braking and free return owing to its photothermogenesis and shape memory abilities. Specifically, as shown in Fig. 5(b), the as-prepared microbot was able to stop traveling when subjected to NIR laser irradiation for approximately $6 \mathrm{~s}$; additionally, it assumed a state where the frictional anisotropy had almost disappeared because the surface had become flexible under NIR irradiation. However, once the NIR light was turned off and the thermal energy dissipated, the microbot began to move again, achieving controllable braking under remote control and in real time (Video S3 in the ESM). Moreover, with the switched surface of $\mathrm{SMP} @ \mathrm{Fe}_{3} \mathrm{O}_{4}$, the microbot easily achieved a round trip, as shown in Fig. 5(c). Under vibrations, the microbot arrived at the destination within $4 \mathrm{~s}$ at an ultrafast speed and was then stopped at the boundary of the rail. Meanwhile, the following $\sim 7 \mathrm{~s}$ of NIR irradiation resulted in the spines resuming to their initial orientation and the microbot turning in the opposite direction directly and moving at a relatively lower speed of $10 \mathrm{~mm} / \mathrm{s}$ (Video S4 in the ESM). The lower speed is attributable to the incomplete recovery of the surface rigidity owing to the short cooling time at the ambient temperature, which might have caused the surface to be in a state of dissipated energy. Compared with the nimble devices available 

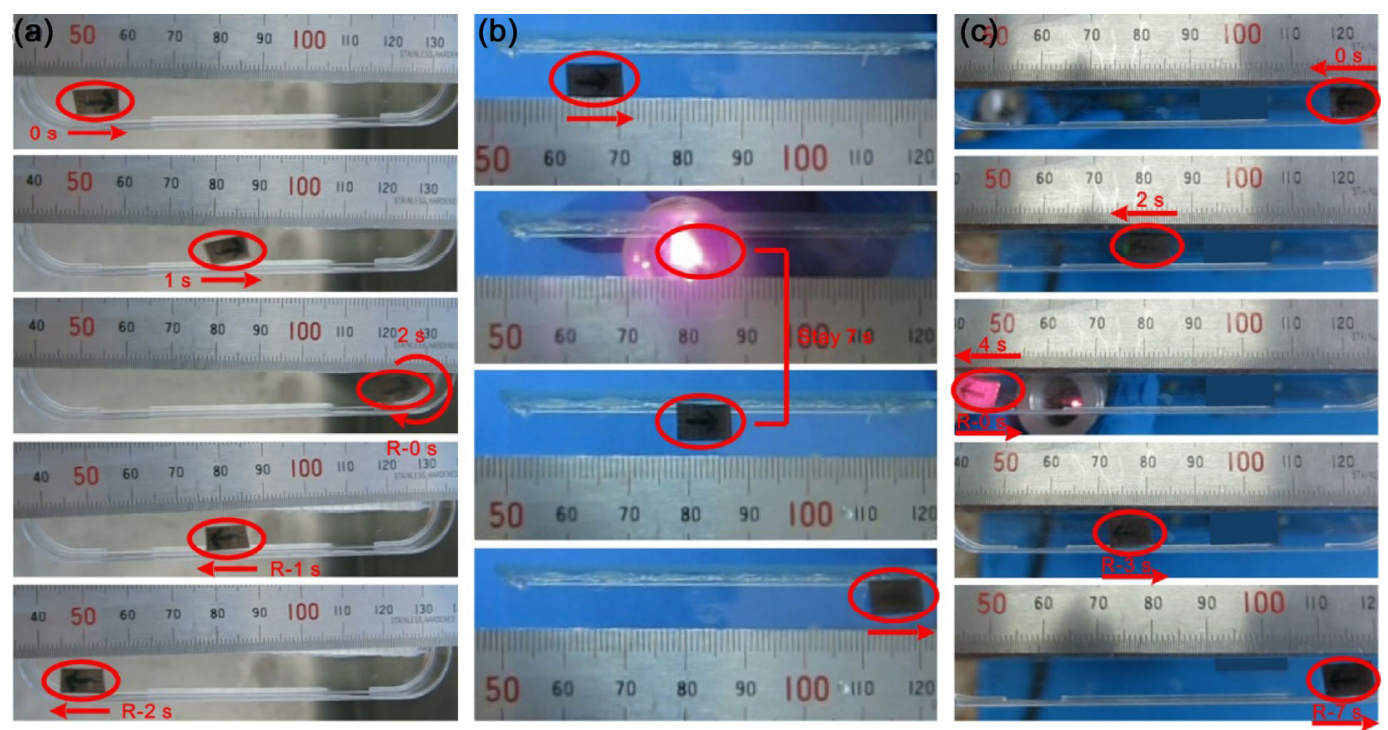

Fig. 5 Images showing locomotive functions of biomimetic device on vibrated platform: (a) directional locomotion; (b) controllable braking; and (c) free returning.

on the market and traditional directional driving systems $[7,9,31,32]$, this microstructured $\mathrm{SMP} @ \mathrm{Fe}_{3} \mathrm{O}_{4}$ device exhibits unique merits, including remote control, braking, and returning with the aid of tweezers. Therefore, we believe that these advantages will enable the further development of controllable directional driving.

\section{Conclusions}

In this study, a smart biomimetic surface was created to mimic anisotropic friction and directional motion. A shape memory polymer containing $\mathrm{Fe}_{3} \mathrm{O}_{4}$ NPs was employed to achieve dynamic friction control and directional driving under NIR irradiation. The friction force can be switched from $0.26 \mathrm{~N}( \pm 0.01 \mathrm{~N})$ to $0.73 \mathrm{~N}$ $( \pm 0.04 \mathrm{~N})$ and from $0.25 \mathrm{~N}( \pm 0.01 \mathrm{~N})$ to $0.59 \mathrm{~N}( \pm 0.03 \mathrm{~N})$ via NIR light-induced changes in the rigidity and orientation, respectively. Moreover, this moving device with shape memory ability not only exhibited superior directionality and fast locomotion speed (with an average value of $\sim 25 \mathrm{~mm} / \mathrm{s}$ ), but also accomplished round-trip movement and braking functions under remote control. These features render the shapememory smart surface highly favorable and broaden its functionality and applications for directional driving, cargo transportation, and areas involving anisotropic friction forces.

\section{Acknowledgements}

The authors gratefully acknowledge the financial support received from the National Natural Science Foundation of China (51775538 and 52005484), the Key Research Program of Frontier Sciences, CAS (QYZDY-SSW-JSC013), and the Oasis Scholar Project of Shihezi University. The authors also thank Dr. Bin LI at Technische Universität München for polishing the language and reviewing the manuscript.

Electronic Supplementary Material Supplementary material is available in the online version of this article at https://doi.org/10.1007/s40544-021-0520-6.

Open Access This article is licensed under a Creative Commons Attribution 4.0 International License, which permits use, sharing, adaptation, distribution and reproduction in any medium or format, as long as you give appropriate credit to the original author(s) and the source, provide a link to the Creative Commons licence, and indicate if changes were made.

The images or other third party material in this article are included in the article's Creative Commons licence, unless indicated otherwise in a credit line to the material. If material is not included in the article's Creative Commons licence and your intended use is not permitted by statutory regulation or exceeds the 
permitted use, you will need to obtain permission directly from the copyright holder.

To view a copy of this licence, visit http://creativecommons.org/licenses/by/4.0/.

\section{References}

[1] Baum M J, Kovalev A E, Michels J, Gorb S N. Anisotropic friction of the ventral scales in the snake lampropeltis getula californiae. Tribol Lett 54(2): 139-150 (2014)

[2] Filippov A E, Westhoff G, Kovalev A, Gorb S N. Numerical model of the slithering snake locomotion based on the friction anisotropy of the ventral skin. Tribol Lett 66(3): 119 (2018)

[3] Song Y, Wang Z Y, Zhou J, Li Y, Dai Z D. Synchronous measurement of tribocharge and force at the footpads of freely moving animals. Friction 6(1): 75-83 (2018)

[4] Gorb S, Scherge M. Biological microtribology: Anisotropy in frictional forces of orthopteran attachment pads reflects the ultrastructure of a highly deformable material. Proc Biol Sci 267(1449): 1239-1244 (2000)

[5] Bauer G, Klein MC, Gorb SN, Speck T, Voigt D, Gallenmüller F. Always on the bright side: The climbing mechanism of Galium aparine. Proc Biol Sci 278(1715): 2233-2239 (2011)

[6] Filippov A, Gorb S N. Frictional-anisotropy-based systems in biology: Structural diversity and numerical model. Sci Rep 3: 1240 (2013)

[7] Ma S H, Scaraggi M, Yan C Y, Wang X L, Gorb S N, Dini D, Zhou F. Bioinspired 3D printed locomotion devices based on anisotropic friction. Small 15(1): 1802931 (2019)

[8] Ji Z, Yan C, Ma S, Zhang X, Jia X, Wang X, Zhou F. Biomimetic surface with tunable frictional anisotropy enabled by photothermogenesis-induced supporting layer rigidity variation. Adv Mater Interfaces 6(2): 1801460 (2019)

[9] Ji Z, Yan C, Ma S, Gorb S, Jia X, Yu B, Wang X, Zhou F. $3 \mathrm{D}$ printing of bioinspired topographically oriented surfaces with frictional anisotropy for directional driving. Tribol Int 132: 99-107 (2019)

[10] Tramsen H T, Gorb S N, Zhang H, Manoonpong P, Dai Z D, Heepe L. Inversion of friction anisotropy in a bio-inspired asymmetrically structured surface. J Roy Soc Interface 15(138): 20170629 (2018)

[11] Wang X, Yang B S, Tan D, Li Q, Song B, Wu Z S, del Campo A, Kappl M, Wang Z K, Gorb S N, et al. Bioinspired footed soft robot with unidirectional all-terrain mobility. Mater Today 35: 42-49 (2020)

[12] Han D, Morde R S, Mariani S, la Mattina A A, Vignali E,
Yang C, Barillaro G, Lee H. 4D printing of a bioinspired microneedle array with backward-facing barbs for enhanced tissue adhesion. Adv Funct Mater 30(11): 1909197 (2020)

[13] Lu H, Zhang M, Yang Y, Huang Q, Fukuda T, Wang Z, Shen Y. A bioinspired multilegged soft millirobot that functions in both dry and wet conditions. Nat Commun 9: 3944 (2018)

[14] Manoonpong P, Petersen D, Kovalev A, Wörgötter F, Gorb S N, Spinner M, Heepe L. Enhanced locomotion efficiency of a bio-inspired walking robot using contact surfaces with frictional anisotropy. Sci Rep 6: 39455 (2016)

[15] Zhang X X, Wang F Y, Yu Y R, Chen G P, Shang L R, Sun L Y, Zhao Y J. Bio-inspired clamping microneedle arrays from flexible ferrofluid-configured moldings. Sci Bull 64(15): 1110-1117 (2019)

[16] Guo Y B, Zhang Z, Zhang S W. Advances in the application of biomimetic surface engineering in the oil and gas industry. Friction 7(4): 289-306 (2019)

[17] Zhou M, Pesika N, Zeng H B, Tian Y, Israelachvili J. Recent advances in gecko adhesion and friction mechanisms and development of gecko-inspired dry adhesive surfaces. Friction 1(2): 114-129 (2013)

[18] Gong W, Zu Y Q, Chen S, Yan Y Y. Wetting transition energy curves for a droplet on a square-post patterned surface. Sci Bull 62(2): 136-142 (2017)

[19] Lv T, Cheng Z J, Zhang D J, Zhang E S, Zhao Q L, Liu Y Y, Jiang L. Superhydrophobic surface with shape memory micro/nanostructure and its application in rewritable chip for droplet storage. ACS Nano 10(10): 9379-9386 (2016)

[20] Cheng Z J, Zhang D J, Lv T, Lai H, Zhang E S, Kang H J, Wang Y Z, Liu P C, Liu Y Y, Du Y, et al. Superhydrophobic shape memory polymer arrays with switchable isotropic/ anisotropic wetting. Adv Funct Mater 28(7): 1705002 (2018)

[21] Han Y, Liu Y X, Wang W X, Leng J S, Jin P. Controlled wettability based on reversible micro-cracking on a shape memory polymer surface. Soft Matter 12(10): 2708-2714 (2016)

[22] Ji Z Y, Yan C Y, Yu B, Wang X L, Zhou F. Multimaterials 3D printing for free assembly manufacturing of magnetic driving soft actuator. Adv Mater Interfaces 4(22): 1700629 (2017)

[23] Yin X, Zhang Y, Wang D, Liu Z, Liu Y, Pei X, Yu B, Zhou F. Integration of self-lubrication and near-infrared photothermogenesis for excellent anti-icing/deicing performance. Adv Funct Mater 25(27): 4237-4245 (2015)

[24] Szyndler M W, Haynes K F, Potter M F, Corn R M, Loudon C. Entrapment of bed bugs by leaf trichomes inspires microfabrication of biomimetic surfaces. $J R$ Soc Interface 10(83): 20130174 (2013) 
[25] Huang S C, Tang Y, Bagheri H, Li D T, Ardente A, Aukes D, Marvi H, Tao J L. Effects of friction anisotropy on upward burrowing behavior of soft robots in granular materials. $A d v$ Intell Syst 2(6): 1900183 (2020)

[26] Gorb E, Gorb S. Contact separation force of the fruit burrs in four plant species adapted to dispersal by mechanical interlocking. Plant Physiol Biochem 40(4): 373-381 (2002)

[27] Zhao Q, Qi H J, Xie T. Recent progress in shape memory polymer: New behavior, enabling materials, and mechanistic understanding. Prog Polym Sci 49-50: 79-120 (2015)

[28] Chen C M, Chiang C L, Lai C L, Xie T, Yang S. Bucklingbased strong dry adhesives via interlocking. Adv Funct Mater 23(30): 3813-3823 (2013)

[29] Liu G, Cai M, Feng Y, Wang X, Zhou F, Liu W.

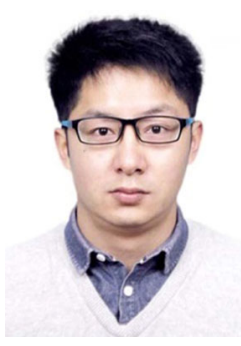

Zhongying JI. He received his B.S. degree in Taishan Medical University, China (2014). He got his Ph.D. degree in 2019 at the Lanzhou Institute of Chemical Physics (LICP), Chinese Academy of Sciences (CAS).

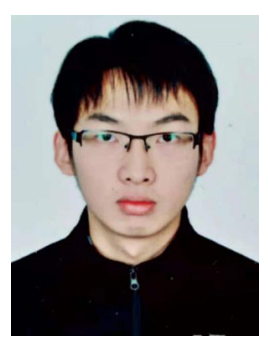

Shiyu QIN. He received his B.S. degree in 2018 from the North China Institute of Science and Technology (NCIST). He has been studying

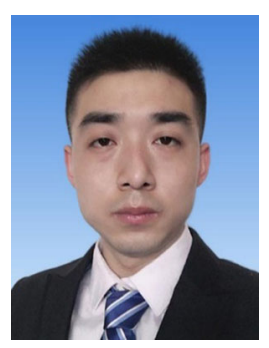

Shuanhong MA. He received his B.S. degree in Tianshui Normal University, China (2011). He got his Ph.D. degree in LICP, CAS (2016). Now, he is an associate professor at the State Key Laboratory of Solid Lubrication, LICP, CAS. He has published over 60 papers, obtained more than 20 patents for invention, and received nearly 1,400 citations. He is a senior member of the
Photothermally actuated interfacial hydration for fast friction switch on hydrophilic polymer brush modified PDMS sheet incorporated with $\mathrm{Fe}_{3} \mathrm{O}_{4}$ nanoparticles. Chem Commun (Camb) 52(18): 3681-3683 (2016)

[30] Yin X, Zhang Y, Lin P, Liu, Wang Z, Yu B, Zhou F, Xue Q. Highly efficient thermogenesis from $\mathrm{Fe}_{3} \mathrm{O}_{4}$ nanoparticles for thermoplastic material repair both in air and underwater. J Mater Chem A 5(3): 1221-1232 (2017)

[31] Dauchot O and Demery V. Dynamics of a self-propelled particle in a harmonic trap. Phys Rev Lett 122(6): 068002 (2019).

[32] Sun L Y, Chen Z Y, Bian F K, Zhao Y J. Bioinspired soft robotic caterpillar with cardiomyocyte drivers. Adv Funct Mater 30(6): 1907820 (2020)

He is now a research assistant at the State Key Laboratory of Solid Lubrication, LICP, CAS. His current research focuses on the $3 \mathrm{D}$ printed functional materials and 3D printed bionic surfaces used for anisotropic friction. His work has been published in more than 20 peer-reviewed papers with nearly 300 citations.

for his M.S. degree since 2018 at Shihezi University (SHZU). His main research interests focus on 3D printing high-performance polymers.

Chinese Mechanical Engineering Society (CMES) and the host of the Young Elite Scientists Sponsorship Program of CAST (2017QNRC0181). He is one of the main inventors for surface catalytic initiated radical polymerization (SCIRP) method in the world. His current research interests focus on the surface wet/ lubricious modification, biolubrication and mechanical bionic tribology (basic research), and product development of lubricious materials \& medical devices (application technology). 


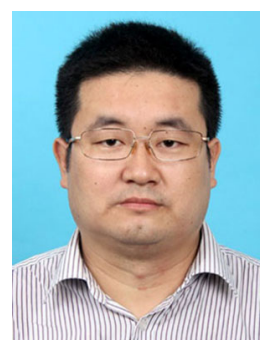

Xin JIA. He is a full professor in SHUZ. He joined the Shihezi University at 2009 after he got his Ph.D. degree in polymer chemistry and physics at Lanzhou University. During his period of Ph.D. study, he spent 18 months (2007-2008) at

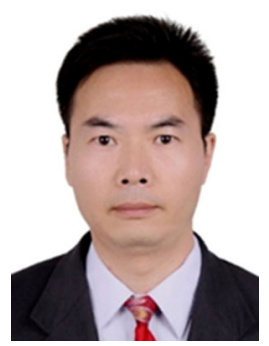

Xiaolong WANG. He is a full professor at the LICP, CAS. He joined the LICP in 2007 after he got his Ph.D. degree with a major in polymer chemistry and physics at Lanzhou University. He spent a year (2010-2011) at Hong Kong

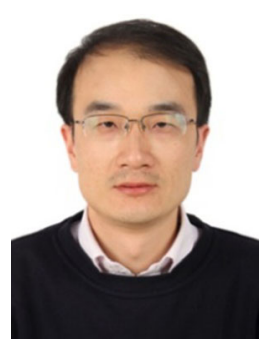

Feng ZHOU. He is a full professor in LICP, CAS, China, and the director of State Key Laboratory of Solid Lubrication, China. He gained his Ph.D. degree in 2004 and spent three years (2005-2008) in the Department of Chemistry, University of Cambridge as a research associate. He has published more than 300 journal papers that received more than 17,000 citations and has the
University of Montreal, Canada, for joint training. His research interests focus on design and application of agricultural functional material, controlled release technology, design and synthesis of functional polymers, and material interface modification. He has published more than 50 articles indexed by SCI.

Polytechnic University as a research associate and two years (2011-2013) at University of Western Ontario, Canada, as an adjunct professor. His research interests focus on 3D printing, biomimetic lubrication, functional soft materials, and flexible electronics. His work has been published in more than 90 peer-reviewed papers with a current h-index of 34 .

high-index of 72 . His research interests include the bioinspired tribology, biomimic surfaces/interfaces of soft matters, drag-reduction and antibiofouling, and functional coatings. He has gained a number of awards including "Outstanding Youth Award" of International Society of Bionic Engineering, 2013, and one National Award for Natural Sciences (the second class). He serves as an editorial board member of Tribology International, Journal of Fiber Bioengineering and Informatics, etc. 